\title{
Étude économique de la production bovine villageoise dans une région du nord de la Côte d'Ivoire infestée par les glossines
}

\author{
P. Itty ${ }^{1}$, G.J. Rowlands ${ }^{2}$, D. Traub ${ }^{3}$, P. Hecker ${ }^{3}$, L. Coulibaly ${ }^{3}$, G. d' Ieteren ${ }^{2}$
}

ITTY (P.), ROWLANDS (G.J.), TRAUB (D.), HECKER (P.), COULIBALY (L.), D'IETEREN (G.). Etude économique de la production bovine villageoise dans une région du nord de la Côte d'Ivoire infestée par les glossines. Revue Élev. Méd. vét. Pays trop., 1994, 47 (3) : 333-343

Cette étude examine la production de troupeaux bovins villageois principalement trypanotolérants, dans la région de Boundiali, au nord de la Côte d'Ivoire. Le but est d'évaluer la rentabilité de ce système de production dans une région affectée par la trypanosomose et d'identifier les principales contraintes économiques. Des données biologiques collectées entre janvier 1986 et décembre 1989 et des données économiques et financières collectées en 1988 sont utilisées dans un Modèle de Troupeau. Ce modèle bio-économique de simulation permet d'obtenir des projections sur 10 ans de la structure des troupeaux, de la production de lait et de viande, et des estimations de rentabilité. Les résultats indiquent une rentabilité économique très élevée pour l'économie ivoirienne. De plus, la récente dévaluation du franc CFA devrait accroître la compétitivité de l'élevage. Ces résultats sont encourageants au vu des efforts du gouvernement pour soutenir l'élevage. La rentabilité finnancière pour les producteurs est en revanche modeste, en particulier en raison des coûts élevés du gardiennage car les bouviers Peul reçoivent une large part du lait produit. Une augmentation de la productivité et de la rentabilité finan. cière paraît difficile tant que subsiste ce système de gestion.

Mots clés : Bovin - Trypanosomose - Glossina - Economie - Rentabilité Analyse coût-bénéfice - Production de viande - Production laitière Méthode d'élevage - Pastoralisme - Côte d'Ivoire.

\section{INTRODUCTION}

La trypanosomose est une contrainte majeure à la production animale en Afrique et l'utilisation de races de bétail trypanotolérant constitue une méthode de contrôle de cette maladie. En 1977, l'Instifut d'Élevage et de Médecine vétérinaire des Pays tropicaux (IEMVT) et le Centre international pour l'Élevage en Afrique (CIPEA) ont constaté qu'il n'y avait que peu de données disponibles sur le bétail trypanotolérant (10). Les études économiques sur le contrôle de la trypanosomose sont également très rares et il n'y a pratiquement rien sur l'économie de la production du bétail trypanotolérant (17). Le Réseau africain d'Étude du bétail trypanotolérant, coordonné par le CIPEA, a été créé afin de combler ces lacunes et d'améliorer la production animale dans les régions infestées par les glossines. Les travaux du Réseau ont déjà contribué à l'élaboration de nouvelles connaissances sur la santé et la productivité des races try-

1. Institut d'Économie rurale, École polytechnique fédérale de Zurich, 8092 Zurich, Suisse.

2. CIPEA/ILCA, POB 46847, Nairobi, Kenya.

3. Projet SODEPRA/GTZ/CIPEA, BP 143, Boundiali, Côte d'Ivoire.

Reçu le 15.6.1993, accepté le 19.10.1994. panotolérantes $(8,16)$. La présente communication s'intègre dans ces travaux en examinant la rentabilité de la production villageoise de bovins principalement trypanotolérants à Boundiali, au nord de la Côte d'Ivoire. Le Département de Boundiali est affecté par la trypanosomose et ce site du Réseau a été établi conjointement avec la Société de Développement de la Production animale (SODEPRA) et la Coopération technique allemande (GTZ).

\section{Description du site}

La Côte 'd'lvoire est traditionnellement importatrice de produits de l'élevage. Il a été estimé qu'en 1991, le pays ne couvrait que 27 p. 100 de ses besoins en viande de boeuf et 15 p. 100 en lait $(12,13)$. Pour faire face à une hausse de la demande due à la croissance démographique et du pouvoir d'achat, le gouvernement a créé, en 1972, la SODEPRA. Le rôle de cette dernière est d'accroître la production locale par un appui aux producteurs. Le troupeau bovin national compte actuellement un peu plus d'un million de têtes dont 86 p. 100 se trouvent dans le nord du pays (9) où le risque trypanosomien est moins élevé. Historiquement, un important noyau d'élevage n'a pu se développer que grâce à la présence de taurins' indigènes trypanotolérants (Baoulé et N'Dama) (7). De nombreux troupeaux Peul se sont installés plus récemment sous l'effet de la sécheresse qui a frappé les pays sahéliens voisins. Ces troupeaux sont constitués principalement de zébus, plus sensibles à la trypanosomose. Cet afflux, en contribuant largement au doublement de la population bovine nationale entre 1975 et 1985, a fortement augmenté la production locale. La population humaine du département de Boundiali est constituée de trois principaux groupes ethniques : les Sénoufo largement majoritaires, les Malinké (20 p. 100 de la population à Boundiali) et les Peul (2). Dans le domaine agricole, les deux premiers groupes s'occupent surtout de cultures, tandis que les Peul, qui ne représentent que $2 \mathrm{p}$. 100 de la population totale, possèdent la moitié des bovins du nord de la Côte d'Ivoire (2). En moyenne, 25 p. 100 des exploitations possèdent des bovins (23).

\section{Le système de production des agriculteurs Sénoufo et Malinké}

Dans le système traditionnel, l'élevage n'est pas considéré comme une activité productive, mais beaucoup plus comme un moyen d'accumulation des surplus dégagés par l'activité essentielle que sont les cultures (11). Cet 
aspect d'épargne reste très important car il y a peu d'autres possibilités d'investissement. Le Sénoufo a deux préoccupations principales par rapport à ses animaux : éviter le vol (préserver son capital) et éviter les dégâts aux cultures (préserver sa source de revenus).

La consommation de viande de boeuf est exceptionnelle en raison du prix élevé. Les adultes consomment très peu ou pas de lait. Le fumier est rarement utilisé et la majorité des paysans ne semble pas en connaître la valeur organique. La traction animale connaît en revanche un essor important même si une large majorité des exploitations cultive encore à la main. Le gardiennage est généralement confié à des bouviers Peul qui traient partiellement les vaches le matin avant de laisser les veaux téter leurs mères. Le lait est vendu par les femmes Peul qui conservent le produit des ventes. En plus des pâturages, les animaux ont accès aux résidus de cultures après les récoltes. La plupart des propriétaires n'affouragent pas de suppléments alimentaires.

\section{Le système de production des pasteurs Peul}

Bien qu'une transhumance partielle soit encore pratiquée durant la saison sèche, les Peul se sédentarisent. La plupart des Peul qui habitent la région utilisent des boeufs de trait pour leurs cultures. On se rapproche d'une exploitation permanente des terres avec rotation entre jachère et cultures et utilisation du fumier (poudrette de parc surtout). Selon BERNARDET (3), les interactions agriculture-élevage et l'intensification de l'agriculture sont plus le fait des Peul que des Sénoufo.

L'afflux de troupeaux Peul depuis les années soixante-dix a intensifié les interactions entre Peul et cultivateurs. Les échanges de lait contre du grain ou de la main-d'oeuvre, l'emploi de Sénoufo dans les champs Peul et les troupeaux Peul pâturant des résidus de cultures des Sénoufo en y déposant du fumier sont des conséquences positives de ces interactions. Du côté négatif, des conflits dus à des dégâts de cultures ont causé mort d'hommes et provoqué le reflux de 50000 bovins Peul vers les pays sahéliens (1). Le gouvernement ivoirien s'efforce d'intégrer les systèmes agraires Peul et Sénoufo afin d'atténuer les conflits et tirer profit des connaissances complémentaires.

\section{MATÉRIEL ET MÉTHODES}

Sept troupeaux villageois ont été choisis pour un suivi mensuel de la santé et de la productivité des bovins. De janvier 1986 à décembre 1989, des données biologiques ont été récoltées sur ces animaux et sur le risque trypanosomien. Les méthodes appliquées sont décrites dans MURRAY et al. (21) et LEAK et al. (20). La détection des trypanosomes dans le sang des bovins a été effectuée au microscope à fond noir par examen du buffy coat. Les troupeaux numérotés de 1 à 5 appartiennent à des
Sénoufo ou Malinké et ceux portant les numéros 6 et 7 appartiennent à des Peul sédentarisés. Au départ, le choix des troupeaux s'est effectué dans le but exclusif d'un suivi biologique. L'accent a porté sur le nombre de bêtes, ce qui explique que trois de ces troupeaux appartiennent à des propriétaires fortunés vivant dans de grandes villes. Comme le système de gestion des troupeaux confiés à la parenté restée au village n'est ni intensif ni particulièrement innovant, on peut présumer que les résultats enregistrés reflètent raisonnablement la situation des troupeaux encadrés par la SODEPRA. II est à relever que les données de la SODEPRA indiquent des performances zootechniques supérieures pour les troupeaux encadrés par rapport à ceux non encadrés (22). En octobre 1988, des données sur les coûts et les prix ainsi que des informations qualitatives sur les systèmes d'élevage ont été collectées pour compléter les données biologiques (17).

\section{Analyses coûts-bénéfices}

Une analyse économique au niveau de la société a été effectuée afin d'évaluer les coûts et les bénéfices des troupeaux pour l'économie ivoirienne. Une analyse financière au niveau privé a été ensuite effectuée afin d'examiner la rentabilité pour les propriétaires de troupeaux. Les valeurs qu'attribuent les individus aux intrants et aux produits diffèrent de la valeur de ces derniers pour l'économie du pays à cause d'imperfections du marché et de l'absence de marché pour certains intrants et produits. Les prix virtuels utilisés pour évaluer les coûts et bénéfices économiques pour la société diffèrent ainsi des prix du marché utilisés pour évaluer les coûts et bénéfices financiers pour les propriétaires. Toutes les valeurs sont données dans la monnaie locale, le Franc de la Communauté financière africaine (F CFA), exprimées en termes constants de 1988. On estime ainsi que l'inflation générale exerce le même effet relatif sur les coûts et les bénéfices. La méthodologie appliquée suit la description de GITTINGER (14).

La performance des entreprises d'élevage est estimée en termes de rentabilité du capital investi. Les critères de rentabilité utilisés sont le ratio bénéfices nets-investissement (RNK) et le taux de rentabilité interne (TRI). Le premier est le résultat de la division de la valeur actualisée des bénéfices nets par la valeur actualisée de l'investissement et le second donne le taux d'intérêt pour lequel la valeur des bénéfices actualisés égale celle des coûts actualisés. Un RNK supérieur à 1 indique que le projet est acceptable car le bénéfice net actualisé excède l'investissement net actualisé. Un TRI supérieur au coût d'opportunité du capital, par exemple 10 p. 100, indique que le projet est acceptable car le taux de rentabilité du capital investi est plus haut que le taux d'intérêt qui peut être obtenu en investissant dans la meilleure alternative.

Les coûts et bénéfices sont projetés sur une période de dix ans en utilisant le Modèle de Troupeau pour Microor- 
dinateur mis au point par le CIPEA (19). C'est un modèle dynamique qui simule sur 10 ans l'évolution du troupeau, sa production et sa performance économique sur la base de valeurs de la structure du troupeau, sa productivité et des données économiques. Il est déterministe du fait que des valeurs moyennes sont utilisées pour la simulation, sans variations aléatoires.

Les données utilisées dans le modèle et leurs calculs sont expliqués ci-dessous. Afin de simplifier la présentation, des références sont aussi faites aux moyennes annuelles des coûts obtenues par les projections du modèle (17).

\section{Données biologiques}

Par rapport à d'autres régions infestées par les glossines, le risque trypanosomien et la prévalence des trypanosomes dans le bétail à Boundiali peuvent être qualifiés de moyens (17). Le risque trypanosomien (nombre de glossines capturées par piège/jour multiplié par le taux d'infection) atteint 8,5. Les espèces capturées sont Glossina palpalis et $G$. tachinoides. La densité apparente des glossines a diminué entre 1986 et 1989 , passant de 3,7 à 0,68 glossines par piège/jour pour $G$. palpalis et de 0,7 à 0,02 glossine par piège/jour pour $G$. tachinoides. Bien que $G$. morsitans soit également présente dans la région, elle n'est capturée que très rarement. La moyenne mensuelle de la prévalence des trypanosomes atteint 11,2 p. 100. Dans une étude antérieure au nord de la Côte d'lvoire, CAMUS (6) a trouvé que malgré un faible taux d'infection des bovins de 6 p. 100, 52 p. 100 des troupeaux étaient contaminés.

Bien qu'il soit reconnu que le bétail fournit d'autres produits et remplit d'autres fonctions, l'attention des auteurs s'est portée sur la production de lait et de viande. Les données mensuelles ont été prises en compte pour calculer les valeurs biologiques de base pour chaque troupeau. Pour estimer les performances du "troupeau moyen", la moyenne arithmétique des valeurs de chaque troupeau individuel a été considérée. La composition phénotypique des troupeaux et les principales valeurs utilisées pour l'analyse sont résumées au tableau I. D'importantes variations de performances apparaissent entre les troupeaux. On note en moyenne une forte mortalité (26 p. 100 jusqu'à un an d'âge, 10 p. 100 de 1 à 2 ans et 8 p. 100 pour les plus de 2 ans), une production modeste de lait prélevé pour la consommation humaine (215 I par lactation) et un taux de reproduction relativement satisfaisant (taux de vêlage 65 p. 100 ; âge au premier vêlage 41 mois). Les taux de vêlage et de mortalité des veaux ont été calculés en incluant les avortements et les mortinatalités.

\section{Données économiques et financières}

Pour l'analyse économique, il a fallu obtenir des estimations du coût d'opportunité du capital investi dans les entreprises d'élevage, du coût des intrants et de la valeur des produits, aussi bien pour l'économie ivoirienne que pour les producteurs individuels. Les tableaux II et III présentent respectivement les moyennes annuelles des valeurs économiques (prix virtuels) et des valeurs financières (prix du marché) des principaux intrants associés à la production de bovins. L'estimation de la valeur des intrants et des produits est expliquée ci-après.

\section{Coût d'opportunité du capital}

Celui-ci est le taux de rentabilité qu'il est possible d'obtenir dans la meilleure alternative. Il a été présumé que ce taux se montait, en termes réels (c.à.d. abstraction faite de l'inflation), à 10 p. 100, aussi bien pour la société que pour les propriétaires de bovins. Comme le critère du TRI est utilisé pour les résultats, les valeurs calculées du TRI peuvent être comparées directement avec toute autre estimation du coût d'opportunité du capital.

\section{Taux de change}

Pour l'analyse financière, le taux de change officiel d'octobre 1988 (300 F CFA pour 1 US\$) a été utilisé. Des estimations de la Banque Mondiale plaçaient la surévaluation du F CFA à 30 p. 100 (14). Le taux virtuel utilisé pour convertir les biens échangés dans l'analyse économique est donc de $429 \mathrm{~F}$ CFA pour 1 US\$.

\section{Coûts de gardiennage}

Selon la taille du troupeau, un ou deux bouviers sont nécessaires. Le coût économique de la main-d'oeuvre dépend des possibilités d'emploi dans la meilleure solution de rechange. Comme le bouvier Peul est une personne peu encline à travailler dans un secteur autre que celui de l'élevage, son coût économique est faible (120 000 F CFA par an). En ce qui concerne les coûts financiers, les bouviers sont payés en espèces (par troupeau et/ou par animal gardé) et en nature (habits, logement, lait). Le tableau IV donne les coûts privés de gardiennage pour chaque troupeau avec la part ou la quantité de lait attribuée séparément. La gestion du troupeau 7 appartenant à une famille Peul est différente des autres car les enfants assurent le gardiennage et ne touchent pas de salaire. La valeur de ce travail est obtenue en estimant la somme à laquelle la famille renonce en employant les enfants pour s'occuper des bêtes, au lieu d'effectuer un autre travail.

\section{Parc de nuit et couloir de contention}

Les troupeaux bénéficient chacun d'un parc et d'un couloir du type SODEPRA. Les coûts économiques sont les suivants : les parcs, qui durent 5 ans, coûtent 96000 F CFA pour des troupeaux de 100 à 200 bêtes et $43000 \mathrm{~F}$ CFA pour des troupeaux comptant 50 à 100 
P. Itty G.J. Rowlands D. Traub P. Hecker L. Coulibaly G. D'Ieteren

TABLEAU I Résumé des données biologiques des troupeaux bovins à Boundiali, Côte d'Ivoire (1986-1989).

\begin{tabular}{|c|c|c|c|c|c|c|c|c|c|}
\hline \multirow{2}{*}{ Rubrique } & \multirow{2}{*}{ Unité } & \multirow{2}{*}{ Moyenne } & \multicolumn{7}{|c|}{ Numéro du troupeau } \\
\hline & & & 1 & 2 & 3 & 4 & 5 & 6 & 7 \\
\hline $\begin{array}{l}\text { Sous-espèces et races }{ }^{*} \\
\text { Trypanotolérant } \times \text { zébu } \\
\text { Baoulé } \\
\text { N'Dama } \\
\text { Zébu }\end{array}$ & $\begin{array}{l}\text { p. } 100 \\
\text { p. } 100 \\
\text { p. } 100 \\
\text { p. } 100\end{array}$ & $\begin{array}{r}41 \\
38 \\
18 \\
3\end{array}$ & $\begin{array}{r}11 \\
57 \\
32 \\
0\end{array}$ & $\begin{array}{r}1 \\
59 \\
41 \\
0\end{array}$ & $\begin{array}{r}43 \\
47 \\
8 \\
1\end{array}$ & $\begin{array}{r}7 \\
57 \\
36 \\
0\end{array}$ & $\begin{array}{r}75 \\
15 \\
0 \\
9\end{array}$ & $\begin{array}{r}88 \\
4 \\
2 \\
6\end{array}$ & $\begin{array}{r}64 \\
24 \\
5 \\
7\end{array}$ \\
\hline $\begin{array}{l}\text { Structure du troupeau } \\
\text { veaux }<1 \text { an } \\
\text { femelles } 1-3 \text { ans } \\
\text { femelles }>3 \text { ans } \\
\text { mâles } 1-3 \text { ans } \\
\text { mâles }>3 \text { ans } \\
\text { Total }\end{array}$ & $\begin{array}{l}\mathrm{Nb} \\
\mathrm{Nb} \\
\mathrm{Nb} \\
\mathrm{Nb} \\
\mathrm{Nb} \\
\mathrm{Nb}\end{array}$ & $\begin{array}{r}32,9 \\
25,1 \\
54,2 \\
19,6 \\
5,5 \\
137,3\end{array}$ & $\begin{array}{r}24,7 \\
15,2 \\
31,9 \\
14,0 \\
2,3 \\
88,1\end{array}$ & $\begin{array}{r}41,0 \\
35,6 \\
61,8 \\
22,9 \\
4,7 \\
166,0\end{array}$ & $\begin{array}{r}52,0 \\
52,8 \\
106,7 \\
25,8 \\
5,8 \\
243,1\end{array}$ & $\begin{array}{r}24,0 \\
14,4 \\
42,3 \\
22,0 \\
6,6 \\
109,3\end{array}$ & $\begin{array}{r}24,8 \\
25,7 \\
50,7 \\
21,4 \\
6,8 \\
129,4\end{array}$ & $\begin{array}{r}25,8 \\
16,0 \\
45,6 \\
19,7 \\
8,2 \\
115,3\end{array}$ & $\begin{array}{r}33,5 \\
23,2 \\
45,7 \\
6,9 \\
1,8 \\
111,1\end{array}$ \\
\hline $\begin{array}{l}\text { Poids vifs } \\
\text { veaux } 1 \text { an } \\
\text { femelles }>3 \text { ans } \\
\text { mâles }>3 \text { ans }\end{array}$ & $\begin{array}{l}\mathrm{kg} \\
\mathrm{kg} \\
\mathrm{kg}\end{array}$ & $\begin{array}{l}100 \\
230 \\
263\end{array}$ & $\begin{array}{l}104 \\
214 \\
284\end{array}$ & $\begin{array}{l}113 \\
233 \\
274\end{array}$ & $\begin{array}{r}91 \\
219 \\
214\end{array}$ & $\begin{array}{r}81 \\
213 \\
222\end{array}$ & $\begin{array}{r}85 \\
222 \\
239\end{array}$ & $\begin{array}{r}95 \\
244 \\
262\end{array}$ & $\begin{array}{l}132 \\
265 \\
347\end{array}$ \\
\hline $\begin{array}{l}\text { Reproduction } \\
\text { taux de vêlage } \\
\text { âge au } 1^{\text {er }} \text { vêllage }\end{array}$ & $\begin{array}{c}\text { p. } 100 \\
\text { mois }\end{array}$ & $\begin{array}{l}65 \\
41\end{array}$ & $\begin{array}{l}82 \\
36\end{array}$ & $\begin{array}{l}74 \\
38\end{array}$ & $\begin{array}{l}43 \\
44\end{array}$ & $\begin{array}{l}66 \\
46\end{array}$ & $\begin{array}{l}56 \\
45\end{array}$ & $\begin{array}{l}65 \\
42\end{array}$ & $\begin{array}{l}66 \\
38\end{array}$ \\
\hline $\begin{array}{l}\text { Production laitière } \\
\text { Taux de mortalité }\end{array}$ & $\mathrm{kg}$ & 215 & 192 & 199 & $215^{\star *}$ & 217 & 234 & $215^{\star *}$ & 233 \\
\hline $\begin{array}{l}0-1 \text { an } \\
1-2 \text { ans } \\
>2 \text { ans }\end{array}$ & $\begin{array}{l}\text { p. } 100 \\
\text { p. } 100 \\
\text { p. } 100\end{array}$ & $\begin{array}{r}26,4 \\
10,4 \\
7,5\end{array}$ & $\begin{array}{r}12 \\
8 \\
7\end{array}$ & $\begin{array}{r}23 \\
3 \\
4\end{array}$ & $\begin{array}{l}33 \\
15 \\
7\end{array}$ & $\begin{array}{r}30 \\
7 \\
3\end{array}$ & $\begin{array}{r}28 \\
5 \\
10\end{array}$ & $\begin{array}{r}31 \\
15 \\
7\end{array}$ & $\begin{array}{l}29 \\
20 \\
14\end{array}$ \\
\hline $\begin{array}{l}\text { Taux d'exploitation } \\
\text { femelles }<1 \text { an } \\
\text { femelles } 1-2 \text { ans } \\
\text { femelles } 2-3 \text { ans } \\
\text { femelles }>3 \text { ans } \\
\text { mâles }<1 \text { an } \\
\text { mâles } 1-2 \text { ans } \\
\text { mâles } 2-3 \text { ans } \\
\text { mâles }>3 \text { ans }\end{array}$ & $\begin{array}{l}\text { p. } 100 \\
\text { p. } 100 \\
\text { p. } 100 \\
\text { p. } 100 \\
\text { p. } 100 \\
\text { p. } 100 \\
\text { p. } 100 \\
\text { p. } 100\end{array}$ & $\begin{array}{r}0,4 \\
-2,9 \\
1,4 \\
6,4 \\
1,3 \\
6,7 \\
32,3 \\
49,4\end{array}$ & $\begin{array}{r}0 \\
-5 \\
0 \\
4 \\
0 \\
17 \\
56 \\
50\end{array}$ & $\begin{array}{r}0 \\
0 \\
0 \\
-2 \\
0 \\
2 \\
52 \\
60\end{array}$ & $\begin{array}{r}3 \\
1 \\
9 \\
19 \\
7 \\
23 \\
58 \\
46\end{array}$ & $\begin{array}{r}0 \\
-8 \\
0 \\
6 \\
0 \\
0 \\
24 \\
52\end{array}$ & $\begin{array}{r}0 \\
-13 \\
-11 \\
9 \\
0 \\
-31 \\
-3 \\
39\end{array}$ & $\begin{array}{r}0 \\
0 \\
4 \\
8 \\
2 \\
3 \\
9 \\
49\end{array}$ & $\begin{array}{r}0 \\
5 \\
8 \\
1 \\
0 \\
33 \\
30 \\
35\end{array}$ \\
\hline
\end{tabular}

* Estimations basées sur l'observation des phenotypes.

** Ces troupeaux n'ont pas été suivis pour la production laitière, les rendements présentés proviennent des valeurs calculées pour le troupeau moyen.

TABLEAU II Coûts économiques des intrants à Boundiali, Côte d'Ivoire (prix virtuels, moyennes annuelles des projections).

\begin{tabular}{|c|c|c|c|c|c|c|c|c|c|}
\hline \multirow{2}{*}{ Rubrique } & \multirow{2}{*}{ Unité } & \multirow{2}{*}{$\begin{array}{c}\text { Troupeau } \\
\text { moyen }\end{array}$} & \multicolumn{7}{|c|}{ Numéro du troupeau } \\
\hline & & & 1 & 2 & 3 & 4 & 5 & 6 & 7 \\
\hline Gardiennage & F CFA/troup. & 171600 & 120000 & 240000 & 240000 & 120000 & 240000 & 120,000 & 120000 \\
\hline Parc et couloir & F CFA/troup. & 65120 & 55920 & 69840 & 69840 & 69840 & 69840 & 69840 & 50700 \\
\hline Services vétérinaires & F CFA/bovin & 610 & 610 & 610 & 610 & 610 & 610 & 610 & 610 \\
\hline Traitements vétérinaires & F CFA/bovin & 2120 & 2160 & 1590 & 2260 & 1510 & 2480 & 2930 & 1890 \\
\hline Production fourragère & F CFA/troup. & 37500 & 0 & 262500 & 0 & 0 & 0 & 0 & 0 \\
\hline Suppléments alimentaires & F CFA/bovin & 90 & 840 & 30 & 0 & 0 & 0 & 0 & 0 \\
\hline Magasin graines coton & F CFA/troup. & 10000 & 70000 & 0 & $\therefore \quad 0$ & 0 & 0 & 0 & 10 \\
\hline Minéraux & F CFA/bovin & 650 & 1050 & 470 & 190 & 1140 & 900 & 550 & 900 \\
\hline
\end{tabular}


TABLEAU III Coûts financiers privés des intrants à Boundiali, Côte d'Ivoire (prix du marché, moyennes annuelles des projections).

\begin{tabular}{|c|c|c|c|c|c|c|c|c|c|}
\hline \multirow{2}{*}{ Rubrique } & \multirow{2}{*}{ Unité } & \multirow{2}{*}{$\begin{array}{c}\text { Troupeau } \\
\text { moyen }\end{array}$} & \multicolumn{7}{|c|}{ Numéro du troupeau } \\
\hline & & & 1 & 2 & 3 & 4 & 5 & 6 & 7 \\
\hline Gardiennage & F CFA/troup. & 761690 & 854550 & 647720 & 1139790 & 584480 & 982980 & 321060 & 120000 \\
\hline Parc et couloir & F CFA/troup. & 21180 & 15960 & 22920 & 22920 & 22920 & 22920 & 22920 & 17700 \\
\hline Traitements vétérinaires & F CFA/bovin & 1290 & 1335 & 946 & 1411 & 878 & 1506 & 1813 & 1174 \\
\hline Production fourragère & F CFA/troup. & 7440 & 0 & 52050 & 0 & 0 & 0 & 0 & 0 \\
\hline Suppléments alimentaires & FCFA/bovin & 54 & 529 & 21 & 0 & 0 & 0 & 0 & 0 \\
\hline Magasin graines coton & F CFA/troup. & 4290 & 30000 & 0 & 0 & 0 & 0 & 0 & 0 \\
\hline Minéraux & F CFAlbovin & 275 & 444 & 200 & 83 & 481 & 382 & 236 & 381 \\
\hline
\end{tabular}

TABLEAU IV Coâts annuels privés de gardiennage par troupeau à Boundiali, Côte d'Ivoire.

\begin{tabular}{|c|c|c|c|c|c|c|c|c|c|}
\hline \multirow{2}{*}{ Rubrique } & \multirow{2}{*}{ Unité } & \multirow{2}{*}{$\begin{array}{l}\text { Troupeau } \\
\text { moyen }\end{array}$} & \multicolumn{7}{|c|}{ Numéro du troupeau } \\
\hline & & & 1 & 2 & 3 & 4 & 5 & 6 & 7 \\
\hline $\begin{array}{l}\text { Taille du troupeau } \\
\text { (moyenne des projections } \\
\text { sur } 10 \text { ans) }\end{array}$ & $\mathrm{Nb}$ & 143 & 98 & 194 & 131 & 119 & 142 & 114 & 98 \\
\hline Nombre de bouviers & $\mathrm{Nb}$ & 1,43 & 1 & 2 & 2 & 1 & 2 & 1 & 1 \\
\hline $\begin{array}{l}\text { Rémunération en espèce } \\
\text { par troupeau }\end{array}$ & F CFA & 313714 & 252000 & 504000 & 648000 & 12000 & 380000 & 280000 & 120000 \\
\hline $\begin{array}{l}\text { par bovin } \\
\text { Rémunération en nature }\end{array}$ & F CFA & 14 & 0 & 0 & 0 & 100 & 0 & 0 & 0 \\
\hline $\begin{array}{l}\text { quantité de lait } \\
\text { part de lait trait }\end{array}$ & $\begin{array}{l}\mathrm{kg} \\
\text { p. } 100\end{array}$ & $\begin{array}{r}219 \\
57\end{array}$ & $\begin{array}{r}0 \\
100\end{array}$ & $\begin{array}{r}1278 \\
0\end{array}$ & $\begin{array}{r}0 \\
100\end{array}$ & $\begin{array}{r}0 \\
100\end{array}$ & $\begin{array}{r}0 \\
100\end{array}$ & $\begin{array}{r}365 \\
0\end{array}$ & $\begin{array}{l}0 \\
0\end{array}$ \\
\hline
\end{tabular}

têtes. Les couloirs, d'une durée moyenne de 3 ans, coûtent $140000 \mathrm{~F}$ CFA. Le troupeau 7 constitue une exception, car le parc a été construit à moindres frais par les propriétaires. Les frais de construction (30000 F CFA, durée 5 ans) sont estimés à l'aide du coût d'opportunité du travail familial et du matériel. Les coûts financiers du parc et du couloir sont très inférieurs aux coûts économiques car la SODEPRA accordait, en 1988, 50 p. 100 de subsides sur le fil barbelé utilisé pour le parc et 78 p. 100 sur les frais du couloir.

\section{Coûts des services vétérinaires}

Des soins vétérinaires étaient prodigués par l'équipe du projet SODEPRA/GTZ/CIPEA afin d'encourager la coopération des propriétaires. Les services réguliers de la SODEPRA sont bien développés et fournissent des prestations similaires. Pour calculer les coûts économiques, les coûts du budget SODEPRA 1988 pour le secteur de Boundiali ont été pris comme base en corrigeant les prix des biens échangés pour la surévaluation du F CFA, et en ajustant les salaires du personnel non qualifié par les coûts d'opportunité du travail. On a présu- mé que les bovins, qui représentent 75 p. 100 des ruminants du secteur, engendrent 90 p. 100 des frais. Le coût annuel ainsi obtenu est de 610 F CFA par bovin. Soixante-neuf p. 100 des coûts concernent les frais de personnel, les 31 p. 100 restants, ceux de transport. Les services vétérinaires ne représentent pas un coût financier pour les propriétaires de bovins car les frais sont pris en charge par l'Etat.

\section{Coûts des traitements vétérinaires}

Ils sont calculés sur la base du prix par traitement pour les médicaments (antibiotiques, Béréni ${ }^{\circledR}$ contre la trypanosomose, acaricides plus pulvérisateur et vermifuges) et les vaccins (peste bovine, péripneumonie, pasteurellose et charbon symptomatique) et assignés aux animaux selon le nombre de traitements et les dosages reçus durant la période du suivi. Les coûts économiques annuels moyens par animal s'élèvent à 2120 F CFA, répartis de la manière suivante : antibiotiques 66 p. 100, Bérénil $^{\circledR} 15$ p. 100 , acaricides 10 p. 100 , vermifuges 7 p. 100 et vaccins 2 p. 100. Les coûts financiers se montent à $1290 \mathrm{~F}$ CFA par animal et par an et sont plus bas en raison des subventions accordées par la SODEPRA. 


\section{P. Itty G.J. Rowlands D. Traub P. Hecker L. Coulibaly G. D'Ieteren}

\section{Production fourragère}

Seul le troupeau 2 bénéficie de pâturages améliorés (12,5 ha établis par la SODEPRA). Les coûts économiques s'élèvent à $2000000 \mathrm{~F} \mathrm{CFA}$ pour l'établissement du pâturage et $62500 \mathrm{~F} \mathrm{CFA/an} \mathrm{pour} \mathrm{l'entretien} \mathrm{(5).} \mathrm{Les} \mathrm{coûts} \mathrm{finan-}$ ciers sont beaucoup plus faibles grâce aux diverses subventions accordées. Ils s'élèvent à $208000 \mathrm{~F}$ CFA pour l'établissement et $31250 \mathrm{~F}$ CFA/an pour l'entretien.

\section{Suppléments alimentaires}

Des graines de coton sont données en supplément aux animaux des troupeaux 1 et 2 . Les coûts économiques annuels se montent à $840 \mathrm{~F}$ CFA/animal pour le troupeau 1 et $30 \mathrm{~F}$ CFA/animal pour le troupeau 2. Du fait de la grande quantité de graine offerte en supplément au troupeau 1, il faut également comptabiliser le coût d'un magasin pour l'entreposage. Le magasin coûte 350000 F CFA et dure 5 ans. Des subventions étant également accordées pour la graine de coton et le magasin, les coûts financiers sont les suivants : troupeau 1, graine de coton $529 \mathrm{~F} \mathrm{CFA} /$ animal/an, magasin $150000 \mathrm{~F} \mathrm{CFA}$ tous les 5 ans et le troupeau 2, $21 \mathrm{~F} \mathrm{CFA/animal/an.}$

\section{Sels minéraux}

Tous les propriétaires donnent des sels minéraux à leurs animaux mais à des degrés variables. Le coût économique annuel moyen est de $650 \mathrm{~F} \mathrm{CFA/bovin} \mathrm{et} \mathrm{le} \mathrm{coût}$ financier $275 \mathrm{~F} \mathrm{CFA/bovin} \mathrm{du} \mathrm{fait} \mathrm{de} \mathrm{subventions.}$

\section{Prix du bétail de reproduction}

Les animaux de reproduction ne sont pas échangés. Leur coût économique est donc égal à leur coût financier (prix du marché) qui est de $300 \mathrm{~F}$ CFA/kg de poids vif (PV).

\section{Prix de la viande de boeuf}

La viande de boeuf est en partie importée et le prix virtuel est donc obtenu en prenant le prix de parité à l'importation. Ce prix est celui du marché mondial corrigé en fonction des frais de transport et de commercialisation (26). Les prix internationaux, exprimés en US\$, sont convertis en FCFA en utilisant le taux de change virtuel. Le prix de la viande de boeuf obtenu se monte à $592 \mathrm{~F} \mathrm{CFA} / \mathrm{kg}$ PV. Le prix du marché pris en compte pour l'analyse financière est nettement inférieur : $300 \mathrm{~F} \mathrm{CFA} / \mathrm{kg} \mathrm{PV}$.

\section{Prix du lait}

Le prix virtuel est calculé similairement au prix du boeuf car la Côte d'Ivoire importe du lait. Le prix de parité à l'importation est de $215 \mathrm{~F} \mathrm{CFA} / \mathrm{kg}$ d'équivalent-lait liquide. Le prix moyen du marché atteint seulement 50 p. 100 du prix virtuel, soit 112,5 F CFA/kg.

\section{Traite et commercialisation du lait}

Dans l'analyse économique, le calcul du prix de parité à l'importation du lait prend en compte la traite et la commercialisation. Dans l'analyse financière, les revenus du lait au seuil de l'exploitation sont calculés en déduisant les frais dus à la traite (par les bouviers) et à la commercialisation du lait (par les femmes des bouviers), du revenu brut (quantité de lait produite multipliée par le prix du marché). Les observations faites ont permis d'estimer le travail nécessaire et d'établir les coûts d'opportunité annuels suivants : $37500 \mathrm{~F}$ CFA par bouvier pour la traite et 30000 F CFA par femme de bouvier pour la commercialisation.

\section{RÉSULTATS}

Des projections sur 10 ans de l'utilisation d'intrants, de la production laitière, des sorties d'animaux et de la structure du troupeau ont été obtenues pour chaque troupeau. Ces projections, combinées avec les paramètres économiques et financiers, fournissent des estimations des coûts et bénéfices et de la rentabilité des entreprises de production bovine.

\section{Analyse économique au niveau de la société}

Les résultats de cette analyse sont présentés dans le tableau V. La rentabilité économique de la production bovine est élevée (TRI moyen de 37 p. 100). La variation des résultats entre les troupeaux est toutefois considérable (TRI variant de 21 p. 100 à 47 p. 100). Les achats de bovins représentent la part majeure des coûts ( 60 p. 100 en moyenne) et les frais médicamenteux sont importants (15 p. 100 en moyenne). Les sorties (ventes, abattages) de bovins représentent 57 p. 100 des revenus tandis que le lait compte pour 31 p. 100 de ceux-ci.

Dans l'analyse, on a pris en compte la surévaluation du F CFA sur la base d'une estimation de $30 \mathrm{p}$. 100. Cette surévaluation s'est accentuée au fil des années, au point que le F CFA a été effectivement dévalué de 50 p. 100 en janvier 1994. Si, en 1988, la surévaluation avait été de 50 p. 100 (taux de change $600 \mathrm{~F}$ CFA pour 1 US\$), la rentabilité économique aurait été encore plus grande : TRI de 49 p. 100 . Le prix virtuel de la viande utilisé pour l'analyse économique est basé sur le prix du marché mondial du boeuf argentin, corrigé pour les frais de transport et de commercialisation (549,9 F CFA/kg PV). Ce calcul peut être discuté car la viande importée au niveau de Boundiali provient essentiellement du Mali. Selon les indications de l'Office malien du Bétail et de la Viande, la moyenne des prix (1988) aux producteurs dans ce pays est de $294 \mathrm{~F} \mathrm{CFA} / \mathrm{kg}$ PV. II est très difficile de calculer les frais de transport et de commercialisation mais même en utilisant la limite inférieure de 300 F CFA/kg PV (26) comme prix de parité à l'importation, la rentabilité économique se maintient à un niveau raisonnable: TRI de 23,1 p. 100. 
TABLEAU V Résultats économiques de la production bovine à Boundiali, Côte d'Ivoire.

\begin{tabular}{|c|c|c|c|c|c|c|c|c|c|}
\hline \multirow{2}{*}{ Rubrique } & \multirow{2}{*}{ Unité } & \multirow{2}{*}{$\begin{array}{c}\text { Troupeau } \\
\text { moyen }\end{array}$} & \multicolumn{7}{|c|}{ Numéro du troupeau } \\
\hline & & & 1 & 2 & 3 & 4 & 5 & 6 & 7 \\
\hline \multicolumn{10}{|l|}{ Coûts } \\
\hline $\begin{array}{l}\text { Coûts annuels } \\
\text { non actualisés/bovin }\end{array}$ & F CFA & 10840 & 12000 & 10530 & 15270 & 9450 & 11080 & 11730 & 12490 \\
\hline Coûts actualisés/bovin & F CFA & 89510 & 94960 & 88280 & 135710 & 76850 & 88380 & 96710 & 106960 \\
\hline \multicolumn{10}{|l|}{$\begin{array}{l}\text { Répartition des coûts } \\
\text { actualisés : }\end{array}$} \\
\hline Achat des bovins & p. 100 & 59,9 & 50,6 & 54,9 & 72,7 & 59,4 & 55,4 & 61,6 & 68,4 \\
\hline Traitements vétérinaires & p. 100 & 15,0 & 14,3 & 11,2 & 11,9 & 12,4 & 17,6 & 19,2 & 11,6 \\
\hline Gardiennage & p. 100 & 8,5 & 8,2 & 8,9 & 8,5 & 8,3 & 12,1 & 6,9 & 7,3 \\
\hline Services vétérinaires & p. 100 & 4,4 & 4,1 & 4,4 & 3,0 & 5,1 & 4,5 & 4,1 & 3,7 \\
\hline Minéraux & p. 100 & 4,5 & 6,8 & 3,3 & 1,0 & 9,1 & 6,3 & 3,6 & 5,4 \\
\hline Parc et couloir & p. 100 & 3,8 & 4,5 & 3,0 & 2,9 & 5,7 & 4,1 & 4,7 & 3,6 \\
\hline $\begin{array}{l}\text { Fourrages et suppléments } \\
\text { alimentaires }\end{array}$ & p. 100 & 3,9 & 11,6 & 14,2 & 0,0 & 0,0 & 0,0 & 0,0 & 0,0 \\
\hline \multicolumn{10}{|l|}{ Revenus } \\
\hline $\begin{array}{l}\text { Revenus annuels } \\
\text { non actualisés/bovin }\end{array}$ & F CFA & 33740 & 43940 & 42850 & 35780 & 32340 & 23570 & 32210 & 33660 \\
\hline Revenus actualisés/bovin & F CFA & 192290 & 253950 & 243240 & 234980 & 182880 & 128910 & 184000 & 196050 \\
\hline \multicolumn{10}{|l|}{$\begin{array}{l}\text { Répartition des revenus } \\
\text { actualisés : }\end{array}$} \\
\hline Sorties de bovins & p. 100 & 56,9 & 62,9 & 64,1 & 75,3 & 58,8 & 44,3 & 56,4 & 50,8 \\
\hline Lait & p. 100 & 31,5 & 28,2 & 26,2 & 20,5 & 29,7 & 38,4 & 30,7 & 38,0 \\
\hline Valeur finale du troupeau & p. 100 & 11,6 & 8,9 & 9,7 & 4,2 & 11,5 & 17,3 & 13,0 & 11,2 \\
\hline Analyse coûts-bénéfices & & & & & & & & & \\
\hline RNK & & 2,74 & 3,87 & 3,52 & 1,97 & 3,16 & 1,77 & 2,38 & 2,16 \\
\hline TRI & p. 100 & 37,3 & 55,5 & 47,2 & 34,7 & 42,5 & 21,4 & 32,7 & 30,4 \\
\hline
\end{tabular}

\section{Analyse financière au niveau privé}

Les résultats financiers privés sont présentés dans le tableau VI. En moyenne, la rentabilité est modeste mais satisfaisante (TRI 11,8 p. 100). Les coûts de gardiennage représentent en particulier une lourde charge (34 p. 100 des coûts en moyenne) et le lait ne contribue qu'à 26 p. 100 des revenus. Le troupeau 2 se distingue par des résultats supérieurs, les troupeaux $1,4,6$ et 7 ont des performances financières modestes, tandis que celles-ci sont clairement insuffisantes pour les troupeaux 3 et 5 .

L'impact d'une dévaluation du F CFA sur la rentabilité pour les producteurs a été examiné. Les résultats indiquent qu'une dévaluation de 50 p. 100 aurait abaissé le taux de rentabilité financière à $9,1 p$. 100 en raison d'un effet direct sur le coût des intrants importés. Suite à la dévaluation, il y a eu également une hausse des prix des produits de l'élevage (15 à 30 p. 100 au premier trimestre 1994 pour le boeuf en Côte d'Ivoire (25)). Pour les troupeaux étudiés, cela aurait dû suffire à maintenir la rentabilité financière au niveau précédant la dévaluation.
Les possibilités de financement des services de vulgarisation par les producteurs sont de plus en plus discutées en Afrique, notamment par la Banque Mondiale. Les coûts des services vétérinaires ont donc été ajoutés dans l'analyse financière. Les résultats montrent que le financement privé réduit le TRI pour les producteurs à 10,6 p. 100 et que si, en plus, les subventions accordées par la SODEPRA pour les autres intrants sont supprimées, la rentabilité atteinte est faible (TRI 8,4 p. 100).

\section{DISCUSSION}

Les troupeaux obtenant les meilleurs résultats zootechniques (troupeaux 1 et 2) ont généré les plus hauts revenus et sont donc les plus rentables. Les taux de reproduction et de survie ont particulièrement contribué à ces performances (tabl. I). Ces deux troupeaux ont bénéficié de fourrages améliorés et/ou de graines de coton. Les troupeaux ayant de faibles performances zootechniques (troupeaux 3 et 7 ) enregistrent une diminution de leur 
TABLEAU VI Résultats financiers de la production bovine à Boundiali, Côte d'Ivoire.

\begin{tabular}{|c|c|c|c|c|c|c|c|c|c|}
\hline \multirow{2}{*}{ Rubrique } & \multirow{2}{*}{ Unité } & \multirow{2}{*}{$\begin{array}{l}\text { Troupeau } \\
\text { moyen }\end{array}$} & \multicolumn{7}{|c|}{ Numéro du troupeau } \\
\hline & & & 1 & 2 & 3 & 4 & 5 & 6 & 7 \\
\hline $\begin{array}{l}\text { Coûts } \\
\text { Coûts annuels }\end{array}$ & & & & & & & & & \\
\hline non actualisés/bovin & FCFA & 12550 & 16350 & 9740 & 20210 & 11040 & 13850 & 11010 & 10290 \\
\hline Coûts actualisés/bovin & F CFA & 98740 & 119950 & 79760 & 166980 & 85320 & 104800 & 91690 & 92500 \\
\hline $\begin{array}{l}\text { Répartition des coûts } \\
\text { actualisés: }\end{array}$ & & & & & & & & & \\
\hline Achat des bovins & p. 100 & 54,3 & 40,0 & 60,8 & 59,1 & 53,5 & 46,7 & 65,0 & 79,1 \\
\hline Gardiennage & p. 100 & 33,6 & 44,9 & 26,3 & 33,8 & 34,8 & 40,9 & 19,3 & 8,4 \\
\hline Traitements vétérinaires & p. 100 & 8,3 & 7,0 & 7,4 & 6,0 & 6,5 & 9,0 & 12,5 & 8,3 \\
\hline Minéraux & p. 100 & 1,7 & 2,3 & 1,5 & 0,3 & 3,5 & 2,3 & 1,6 & 2,6 \\
\hline Parc et couloir & p. 100 & 1,1 & 1,0 & 1,1 & 0,8 & 1,7 & 1,1 & 1,6 & 1,5 \\
\hline $\begin{array}{l}\text { Fourrages et suppléments } \\
\text { alimentaires }\end{array}$ & p. 100 & 0,8 & 4,8 & 2,8 & 0,0 & 0,0 & 0,0 & 0,0 & 0,0 \\
\hline $\begin{array}{l}\text { Revenus (1) } \\
\text { Revenus annuels }\end{array}$ & & & & & & & & & \\
\hline non actualisés/bovin & F CFA & 19430 & 24670 & 24220 & 18480 & 18660 & 13990 & 18940 & 19370 \\
\hline Revenus actualisés/bovin & FCFA & 105260 & 136800 & 131710 & 118400 & 100440 & 71340 & 102320 & 107150 \\
\hline $\begin{array}{l}\text { Répartition des revenus } \\
\text { actualisés: }\end{array}$ & & & & & & & & & \\
\hline Sorties de bovins & p. 100 & 52,7 & 59,1 & 60,0 & 75,8 & 54,2 & 40,5 & 51,4 & 47,1 \\
\hline Lait & p. 100 & 26,2 & 24,3 & 22,1 & 15,9 & 24,8 & 28,1 & 25,3 & 32,4 \\
\hline Valeur finale du troupeau & p. 100 & 21,1 & 16,6 & 18,0 & 8,3 & 20,9 & 31,3 & 23,3 & 20,5 \\
\hline Analyse coûts-bénéfices & & & & & & & & & \\
\hline RNK & & 1,12 & 1,33 & 2,01 & 0,52 & 1,32 & 0,41 & 1,17 & 1,20 \\
\hline TRI & p. 100 & 11,8 & 15,0 & 24,6 & $-3,2$ & 14,9 & 0,2 & 12,8 & 13,5 \\
\hline
\end{tabular}

(1) Déduction faite du cout d'oppontunité de la traite et de la commercialisation du lait.

taille durant les 10 ans projetés (tabl. I, IV). Ceci a pour résultat un investissement important par bovin pour l'achat du troupeau (tabl. V, VI). Le troupeau 3 procure des revenus relativement hauts en dépit de résultats zootechniques médiocres. Ceci est dû, entre autres, à la cadence d'exploitation élevée qui permet d'obtenir des revenus importants grâce à la rapidité de génération de bénéfices (majeure partie des revenus provenant des sorties de bovins). Cette rapidité est un facteur déterminant à cause du procédé d'actualisation appliqué aux analyses coûts-bénéfices. Cette exploitation intensive se fait toutefois au détriment du capital de production (le troupeau de reproduction), ce qui a pour conséquence une rentabilité inférieure. Cette discussion montre qu'une augmentation de la productivité biologique, reproduction et survie en premier lieu, a un effet considérable sur la rentabilité.

Par rapport aux autres sites couverts par l'étude plus exhaustive (17), on note que les traitements vétérinaires représentent une lourde charge, non seulement par rap- port aux coûts totaux (15 p. 100 des coûts économiques et 8 p. 100 des coûts financiers) mais aussi en valeur absolue. Durant le suivi, le projet a fourni gratuitement les médicaments pour les sept troupeaux à 'titre de dédommagement et d'incitation à participer à l'étude. Du fait de cette gratuité des soins, il est possible que cette demande de médicaments ait été plus forte que la moyenne dans la région. II faut donc relativiser quelque peu l'importance de ces coûts telle qu'elle apparaît ici dans les résultats.

La forte rentabilité économique (tabl. V) est encourageante au vu des efforts déployés par le gouvernement pour soutenir l'élevage local. Une des raisons de cette rentabilité tient au faible coût d'opportunité du travail des pasteurs, qui engendre des frais de gardiennage peu élevés si on les compare à d'autres sites africains (17). La raison première est toutefois la surévaluation élevée du F CFA qui renchérit les: prix virtuels des biens échangés. Cette rentabilité économique, qui se trouve accrue par une augmentation de la surévaluation du F CFA, illustre 
un des effets escomptés d'une dévaluation accompagnée d'une libéralisation des prix. Ces mesures devraient relancer les secteurs produisant des biens échangés, tels que le boeuf et le lait, du fait d'une amélioration de leurs prix et de leur compétitivité internationale $(25,26)$. Suite à la dévaluation, le lait et la viande importés d'outre-mer coûtent plus cher, ce qui augmente la demande relative pour le lait et la viande du pays et de la zone CFA. Les analyses de sensitivité suggèrent que la rentabilité financière n'a probablement pas été fortement affectée par la dévaluation. Celle-ci est toutefois trop récente pour qu'on puisse en tirer des enseignements définitifs (25). Les limites du développement des filières locales et les effets de l'inflation, notamment sur les prix des productions et leur consommation, exercent également, en effet, des influences déterminantes sur l'économie de l'élevage.

La rentabilité financière des troupeaux est moindre par rapport à la rentabilité économique. Elle est causée par une combinaison de coûts plus élevés et de revenus plus bas (tabl. Vl). Ceci suggère qu'un examen des résultats est indiqué car il pourrait révéler des contraintes importantes sur le plan financier privé. Bien que les intrants soient subventionnés et que l'utilisation du taux de change officiel diminue le prix des intrants importés, les coûts financiers actualisés sont plus élevés que les coûts économiques. Cela provient des coûts privés de gardiennage qui sont, en moyenne, plus de quatre fois supérieurs aux coûts économiques (761 690 F CFA contre $171600 \mathrm{~F} \mathrm{CFA/troupeau/an).} \mathrm{Les} \mathrm{frais} \mathrm{de}$ gardiennage déterminent largement le niveau total des coûts. Les troupeaux 2, 6 et 7 ont des coûts totaux modérés car les bouviers ne bénéficient pas de la totalité du produit de la traite. La participation des bouviers aux bénéfices (lait) s'apparente au métayage. Les hypothèses suivantes pourraient expliquer ce système (17, 24). Les propriétaires possèdent des bovins, non pas pour le lait mais en premier lieu comme investissement. II y a asymétrie d'information car les Peul ont une bien meilleure connaissance de la gestion du bétail. Le lait donné aux bouviers leur fournit une incitation à effectuer un travail de qualité malgré le fait qu'une étroite surveillance des bouviers ne soit possible. Contrairement aux propriétaires Peul, les propriétaires Sénoufo et Malinké ne considèrent pas le lait attribué aux bouviers comme un coût (la demande de lait frais par les adultes Sénoufo et Malinké est faible). Si ce système de gestion est certainement logique, il représente une contrainte importante à une augmentation de la productivité bovine et de la rentabilité pour les producteurs. En effet, ceux-ci ne bénéficiant pas de la totalité des gains résultant d'une hausse de la productivité, ils ne sont pas nécessairement encouragés à effectuer des investissements dans ce sens. Ces résultats n'ont pas seulement des implications pour la Côte d'Ivoire, mais également pour de nombreuses régions africaines, en particulier en bordure du Sahel, où le système de gestion est semblable $(4,18)$.
Les analyses simulant la participation des propriétaires aux frais des services vétérinaires ainsi qu'à la prise en charge des subsides accordés par la SODEPRA devraient inciter à une réflexion sur la durabilité de systèmes basés sur une large prise en charge des coûts par l'État. Les revenus financiers ne représentent que 57 p. 100 des revenus économiques (19 430 F CFA contre $33740 \mathrm{~F}$ CFA/bovin en moyenne) pour les valeurs non actualisées (tabl. V et Vl). Ceci est dû aux prix du marché du lait $(112,5 \mathrm{~F} \mathrm{CFA} / \mathrm{kg})$ et de la viande $(300 \mathrm{~F}$ $\mathrm{CFA} / \mathrm{kg}$ ) très inférieurs aux prix virtuels (respectivement $215 \mathrm{~F} \mathrm{CFA} / \mathrm{kg}$ et $592 \mathrm{~F} \mathrm{CFA} / \mathrm{kg}$ ). Les prix des importations de lait et de viande ont été maintenus à des niveaux artificiellement bas à cause de la surévaluation du F CFA et des subsides à l'exportation accordés par l'Union Européenne pour leurs excédents. Ceci a contribué à exercer une pression vers le bas sur les prix de ces produits en Côte d'Ivoire $(25,26)$.

\section{CONCLUSION}

Cette étude montre que l'élevage de bétail principalement trypanotolérant dans cette région infestée par les glossines est rentable. Le système de production examiné à Boundiali est particulièrement rentable du point de vue de l'économie ivoirienne. Ces résultats sont, de plus, extrêmement stables par rapport à diverses hypothèses examinées. Les résultats financiers au niveau privé révèlent une rentabilité aux producteurs en moyenne satisfaisante mais indiquent d'importantes contraintes. Les profits générés sont modestes et la marge de manoeuvre pour des investissements est réduite. Les différences de performances zootechniques entre les troupeaux expliquent largement la grande variabilité des résultats. Cela montre dans quelle mesure une amélioration de la productivité biologique conduit à une rentabilité supérieure.

La contrainte majeure à un accroissement de la production bovine est apparente lorsque l'on compare les analyses économiques et financières. La rentabilité financière modeste est principalement due aux coûts élevés de gardiennage. Ceux-ci sont dus au système de gestion des troupeaux séparant la propriété (les propriétaires sont en général des cultivateurs) du gardiennage (effectué par les pasteurs). II est difficile d'améliorer la productivité du système tant que subsiste le système de paiement des bouviers qui bénéficient du lait produit. Seule une partie de la production revient en effet aux propriétaires.

La récente dévaluation du F CFA devrait relancer le secteur de l'élevage qui, d'une part, fournit du lait et de la viande maintenant plus compétitifs par rapport aux produits importés d'outre-mer et, d'autre part, utilise surtout des intrants locaux dont les prix ne sont que peu affectés par cette mesure économique. Considérant ces développements, l'avenir de l'élevage au nord de la Côte d'Ivoire apparaît donc prometteur. 


\section{P. Itty G.J. Rowlands D. Traub P. Hecker L. Coulibaly G. D'Ieteren}

\section{REMERCIEMENTS}

Notre gratitude va aux agriculteurs et aux bouviers de Boundiali pour leur collaboration lors de la collecte des données et au personnel de la SODEPRA-Nord pour l'appui accordé lors du travail de terrain. Nous aimerions également remercier $P$. Rieder de l'Institut d'Économie rurale de l'École polytechnique fédérale de Zurich, les membres actuels et précédents du Réseau africain d'Étude du Bétail trypanotolérant, $S$. Nagda et $M$. Rarieya en particulier, pour leur appui au projet de recherche. Nous sommes reconnaissants à J. Mclntire sans qui l'étude économique n'aurait pas été initiée et à $S$. Sandford et T. Williams pour leurs suggestions. Nous remercions également les trois lecteurs anonymes pour leurs commentaires.

\section{BIBLIOGRAPHIE}

1. ACKAH (A.P.). Opération d'encadrement des éleveurs du Nord. Rapport annuel 1987. Korhogo, Côte d'Ivoire, Ministère de la Production animale, SODEPRA-Nord, 1988.

2. BAKAYOKO (K.). Rapport trimestriel Janvier-Mars 1988. SODEPRA Nord, zone de Boundiali. Boundiali, Côte d'Ivoire, Ministère de la Production animale, 1988.

3. BERNARDET (P.). Association' agriculture élevage en Afrique. Les Peul semi-transhumants de Côte d'Ivoire. Paris, France, Editions l'Harmat$\tan , 1984.236 \mathrm{p}$

4. BRANDL (F.E.). Economics of trypanosomiasis control in cattle. Kiel, Allemagne, Wissenschaftsverlag, 1988. 220 p.

5. BURHIN (M.), DJIBRIL (M.). Dynamique et rentabilité du pâturage artificiel sous fortes charges avec différents systèmes de production bovine dans le nord de la Côte d'Ivoire. Premiers résultats. Korhogo, Côte d'Ivoire Ministère de la Production animale, SODEPRA-nord,1988. $10 \mathrm{p}$

6. CAMUS (E.). Epidémiologie et incidence clinique de la trypanosomose bovine dans le nord de la Côte d'Ivoire. Perspectives d'avenir en fonction de la diffusion croissante du sang zébu. Revue Élev. Méd. vét. Pays trop., 1981,34 (3) : 289-295.

7. CAMUS (E.), LANDAIS (E.), POIVEY (J.P.). Structure génétique du cheptel bovin sédentaire du nord de la Côte d'Ivoire. Perspectives d'avenir en fonction de la diffusion croissante du sang zébu. Revue Élev. Méd. vét. Pays trop.., 1981, 34 (2) : 187-198.

8. CIPEA. Production animale dans les régions d'Afrique infestées par les glossines. Compte rendu d'une réunion organisée à Nairobi (Kenya) du 23 au 27 novembre 1987. Nairobi, Kenya, CIPEA, 1988. 530 p.

9. Côtc d'Ivoire. Programme de développement de nouveaux systèmes de production (élevages bovins et ovins), rapport d'identification. Abidjan, Côte d'Ivoire, Ministère de la Production Animale, Direction et Contrôle des Grands Travaux. 1988.

10. COULOMB (J.), GRUVEL (J.), MOREL (P.C.) et al. La trypanotolérance : synthèse des connaissances actuelles. Maisons-Alfort, IEMVT, Paris, Ministère de la Coopération, 1977.277 p
11. ESCAFRE (A.). Projet d'aménagements pastoraux. Korhogo, Côte d'Ivoire, SODEPRA-Nord, 1983.

12. FAO. Annuaire de la production. 1992

13. FAO. Annuaire du commerce. 1992.

14. GITTINGER (J.P.). Analyse économique des projets agricoles. 2e edn. Institut de Développement économique de la Banque Mondiale. Paris, France, Fditions Economica, 1985

15. HUBAND (M.). Cost to Paris of African franc zone fuels devaluation debate. The Financial Times, 21st March 1990: 4

16. ILRAD/ILCA. Proceedings of Workshop Towards Increased Utilisation and Adoption of Trypanotolerance. Current Status and Future Directions. Nairobi, Kenya, ILRAD/ILCA, 1993.

17. ITTY (P.). Economics of village cattle production in tsetse affected areas of Africa. A study of trypanosomiasis control using trypanotolerant cattle and chemotherapy in Ethiopia, Kenya. The Gambia, Côte d'Ivoire, Zaire and Togo. Constance, Allemagne, Hartung-Gorre Verlag, 1992 $316 \mathrm{p}$.

18. ITTY (P.), SWALLOW (B.M.), ROWLANDS (G.J.), AGYEMANG (K.), DWINGER (R.H.). Economics of village production of N'Dama cattle in The Gambia. Q. J. int. Agric., 1993, 32 (3) : 293-307.

19. KAUFMANN (Von R.), McINTIRE (J.), ITTY (P.). ILCA Bio-Economic Herd Model for Microcomputer (IBIEHM). User's Manual and Technical Reference Guide. Addis Abeba, Ethiopie, CIPEA, 1990. $38+$ $65 \mathrm{p}$.

20. LEAK (S.G.A.), AWOUME (K.), COLARDELLE (C.) DUFFERA(W.), FERON (A.), MAHAMAT (B.), MAWUENA (K,) MINENGU (M.), MULUNGO (M.), NANKODABA (G.), ORDNER (G.), PELO (M.), SHERIA (M.), TIKUBET (G.), TOURÉ (M.). Détermination de la pression glossinaire et ses relations avec la prévalence des trypanosomes dans le bétail trypanotolérant dans les sites du Réseau africain d'étude du bétail trypanotolérant. In: Production animale dans les régions d'Afrique infestées par les glossines. Compte rendu de réunion 23-27 novembre 1987. Nairobi, Kenya. CIPEA/ILRAD, 1988. p. 48-60.

21. MURRAY (M.), TRAIL (J.C.M.), TURNER (D.A.), WISSOCQ (Y.) Productivité et trypanotolérance. Manuel de formation pour les activités du Réseau. Addis Abeba, Ethiopie, CIPEA, 1983. 221 p.

22. ROBINET (O.). L'élevage bovin dans la zone de savane de Côte d'Ivoire. Concurrence et complémentarités avec la culture cotonnière. Mémoire DESS. Maisons Alfort, France, IEMV'I', 1987. 194 p.

23. SCHUETTERLE (A.), COULIBALY (M.). Aspects socio-économiques de la production du bétail au nord de la Côte d'Yvoire. In: Production animale dans les régions d'Afrique infestées par les glossines. Compte rendu de réunion 23-27 novembre 1987. CIPEA/ILRAD, Nairobi, Kenya. 1988. p. $435-446$

24. SINGH (N.). Theories of sharecropping. In: BARDHAN (P.) ed. The economic theory of agrarian institutions. Oxford, Royaume Uni, Clarendon press,.1989. p. 33-72

25. SOLAGRAL. Bétail et viandes en Afrique de l'Ouest et du Centre. Paris, France, Solagral, Réseau Stratégies Alimentaires, 1994.

26. WILLIAMS (T.O.). Livestock pricing policy in sub-Saharan Africa: objectives, instruments and impact in five countries. Agric. Econ., 1993, 8: $139-159$. 
ITTY (P.), ROWLANDS (G.J.), TRAUB (D.), HECKER (P.), COULIBALY (L.), D'IETEREN (G.). Economic study of village cattle production in a Glossina-infested area in northern Côte d'Ivoire. Revue Elev. Méd. vét. Pays trop., 1994, 47 (3): 333-343

This study examines the production of predominantly trypanotolerant village cattle herds under risk of trypanosomosis in Boundial area, northern Côte d'Ivoire. The aim is to estimate returns and identify the major economic constraints. Biological data collected between January 1986 and December 1989 and economic and financial data collected in 1988 are used in a Herd Model. 10-year projections of herd structure, meat and milk production and returns were obtained from this bio-economic herd simulation model. The results reveal very high economic returns to the country's economy. 'The recent devaluation of the F CFA should in addition increase the competitiveness of the livestock sector. These results are positive in view of the government's efforts to enhance livestock production. The financial returns to the producers are, however, modest, particularly because of high herding costs as the Peul herdsmen receive a large share of the milk produced. Increased productivity and financial returns appear difficult to achieve under this management system.

Key words: Cattle - Trypanosomosis - Glossina - Economics - Profitability Cost profit analysis - Meat production - Milk yield - Animal husbandry method - Pastoralism - Côte d'Ivoire.
ITTY (P.), ROWLANDS (G.J.), TRAUB (D.), HECKER (P.), COULIBALY (L.), D'IETEREN (G.). Estudio económico de la producción bovina aldeana en una región del norte de Côte d'Ivoire infestada por glosinas. Revue Élev. Méd. vét. Pays trop., 1994, 47 (3) : 333-343

Este estudio examina la producción de los hatos bovinos a nivel aldeano, principalmente tripano-tolerantes, en la región de Boundiali, al norte de Côte d'Ivoire. El objetivo es el de evaluar la rentabilidad de este sistema de producción en una región afectada por la tripanosomosis, así como la identificación de los principales obstáculos económicos. Los datos biológicos, colectados entre enero 1986 y diciembre 1989 y los datos económicos y financieros, colectados en 1988, se utilizan en un Modelo de Hato. Este modelo bio-económico de simulación, permite obtener proyecciones a 10 años de la estructura del hato, de la producción de leche y de carne, así como estimaciones de la rentabilidad. Los resultados indican una rentabilidad económica muy elevada para la economía del país. La reciente devaluación del franco CFA, debería además aumentar la competitividad de la crianza. Los resultados son alentadores considerando los esfuerzos gubernamentales para impulsar la crianza. Ia rentabilidad financiera para los productores es, en cambio, modesta, debido en primer lugar, a los elevados costos de cuido, ya que los vaqueros Peul reciben una gran parte de la leche producida. Mientras se mantenga este sistema de manejo, un aumento en la productividad y en la rentabilidad financiera parece dificil.

Palabras clave : Bovino - Tripanosomosis - Glossina - Economía - Rentabilidad - Análisis de costo y beneficio - Producción de carne - Producción lechera - Método de crianza - Pastoralismo - Côte d'Ivoire. 